\title{
Accurate transfer of bimaxillary orthognathic surgical plans using computer-aided intraoperative navigation
}

\author{
Chen Chen ${ }^{\mathrm{a}}$ (1) \\ Ningning Sun ${ }^{\mathrm{b}}$ \\ Chunmiao Jiang ${ }^{\mathrm{c}}$ \\ Yanshan $\mathrm{Liu}^{\mathrm{a}}$ \\ Jian Sun ${ }^{\mathrm{a}, \mathrm{d}}$ (1)
}

${ }^{a}$ Department of Oral and Maxillofacial Surgery, The Affiliated Hospital of Qingdao University, Qingdao, Shandong, China

${ }^{b}$ Department of Emergency Intensive Care Unit, The Affiliated Hospital of Qingdao University, Qingdao, Shandong, China

'Department of Orthodontics, The Affiliated Hospital of Qingdao University, Qingdao, Shandong, China

${ }^{\mathrm{d}}$ Dental Digital Medicine \& 3 D Printing Engineering Laboratory of Qingdao University, Qingdao, Shandong, China
Objective: To examine the accuracy of computer-aided intraoperative navigation (Ci-Navi) in bimaxillary orthognathic surgery by comparing preoperative planning and postoperative outcome. Methods: The study comprised 45 patients with congenital dentomaxillofacial deformities who were scheduled to undergo bimaxillary orthognathic surgery. Virtual bimaxillary orthognathic surgery was simulated using Mimics software. Intraoperatively, a Le Fort 1 osteotomy of the maxilla was performed using osteotomy guide plates. After the Le Fort 1 osteotomy and bilateral sagittal split ramus osteotomy of the mandible, the mobilized maxilla and the distal mandibular segment were fixed using an occlusal splint, forming the maxillomandibular complex (MMC). Realtime Ci-Navi was used to lead the MMC in the designated direction. Osteoplasty of the inferior border of the mandible was performed using Ci-Navi when facial symmetry and skeletal harmony were of concern. Linear and angular distinctions between preoperative planning and postoperative outcomes were calculated. Results: The mean linear difference was $0.79 \mathrm{~mm}$ (maxilla: $0.62 \mathrm{~mm}$, mandible: $0.88 \mathrm{~mm}$ ) and the overall mean angular difference was $1.20^{\circ}$. The observed difference in the upper incisor point to the Frankfort horizontal plane, midfacial sagittal plane, and coronal plane was $<1 \mathrm{~mm}$ in 40 cases. Conclusions: This study demonstrates the role of $\mathrm{Ci}-\mathrm{Navi}$ in the accurate positioning of bone segments during bimaxillary orthognathic surgery. Ci-Navi was found to be a reliable method for the accurate transfer of the surgical plan during an operation.

[Korean J Orthod 2021;51(5):321-328]

Key words: Orthognathic surgery, Virtual surgery planning, Computer-assisted surgery, Intraoperative navigation

Received October 27, 2020; Revised February 9, 2021; Accepted February 15, 2021.

Corresponding author: Jian Sun.

Professor, Department of Oral and Maxillofacial Surgery, The Affiliated Hospital of Qingdao University, No.16 Jiangsu Road, Qingdao 266000, Shandong, China.

Tel +86-0532-82911359 e-mail sunjianqy@126.com

How to cite this article: Chen C, Sun N, Jiang C, Liu Y, Sun J. Accurate transfer of bimaxillary orthognathic surgical plans using computer-aided intraoperative navigation. Korean J Orthod 2021;51:321-328.

(C) 2021 The Korean Association of Orthodontists.

This is an Open Access article distributed under the terms of the Creative Commons Attribution Non-Commercial License (http://creativecommons.org/licenses/by-nc/4.0) which permits unrestricted non-commercial use, distribution, and reproduction in any medium, provided the original work is properly cited. 


\section{INTRODUCTION}

Accurate treatment planning is essential for obtaining optimum esthetic and functional results. ${ }^{1,2}$ Conventional treatment planning using dental plaster casts is unlikely to ensure the anatomical conformation of the overall skull and can lead to a range of unforeseen consequences. ${ }^{3,4}$ The procedure is complex and time-consuming for both patients and doctors. ${ }^{5}$ Furthermore, as model surgery is performed manually, errors are inevitable.

Computer-aided surgical planning (CASP) has been increasingly implemented in orthognathic surgery. ${ }^{3,6,7}$ Advantages of CASP over the conventional method have been previously reported. ${ }^{2,8}$ Accurate transfer of the surgical plan during an operation is important. Due to the mobility and autorotation of the mandible, uneven manual compression by the surgeon occurs on both sides during the repositioning of down-fractured maxillae (particularly when dealing with asymmetric cases); transferring the virtual plan via the traditional intermediate splint often leads to misalignment during maxilla repositioning. These consequences are difficult to detect and promptly correct during the operation. Recently, a series of maxillary reposition templates, independent of the mandible, yielded satisfactory results. ${ }^{7,9-11}$ However, the timely detection of maxilla deviation during surgery remains a challenging task.

Computer-aided intraoperative navigation (Ci-Navi) is a novel method of surgical deviation detection and guidance during an operation. ${ }^{12,13}$ It enables immediate visualization of the surgical plan; the surgeon can make appropriate intraoperative adjustments based on the immediate surgical outcome. This is highly beneficial for the intraoperative real-time guidance of jaw repositioning in orthognathic surgery. However, studies on the application of Ci-Navi in orthognathic surgery are scarce and have only been conducted using small samples. ${ }^{14,15}$

The study aimed to analyze the differences between the preoperative plan and actual postsurgical outcome, and thereby evaluate the accuracy of Ci-Navi in surgical plan transfer during bimaxillary orthognathic surgery.

\section{MATERIALS AND METHODS}

\section{Patients}

This study was designed in compliance with the Declaration of Helsinki, and approval was obtained from the Ethics Committee of the Affiliated Hospital of Qingdao University (No. QYFYWZLL25541). Informed consent was obtained from all the patients before participation.

Adult patients with skeletal malocclusion who were scheduled to undergo bimaxillary orthognathic surgery at the maxillofacial department of the Affiliated Hospital of Qingdao University between July 2018 and August
2019 were enrolled. The inclusion criteria were: (1) adult patients with congenital dentomaxillofacial deformities who were scheduled to undergo bimaxillary orthognathic surgery, and (2) the surgery was assisted by Ci-Navi. The exclusion criteria were: (1) patients with cleft lip/ palate; (2) patients with skeletal deformities owing to trauma or tumor resection; and (3) patients requiring single jaw surgery.

A total of 45 patients ( 22 men, 23 women, average age 23.2 years, range 17-37) were selected for the study. Of these, 37 patients were diagnosed with Class 111 malocclusion, five patients with Class 11 , and three patients with Class 1. Facial asymmetry and/or malformation were diagnosed in 23 patients. Additionally, eight patients had an anterior open bite. In 24 cases, osteoplasty of the inferior border of the mandible was performed to achieve symmetry of the lower face.

\section{Computer-aided surgical planning}

High-resolution computed tomography (CT) examination (Discovery CT750HD; GE Healthcare, Milwaukee, WI, USA) was performed in the preoperative period and 1 month postoperatively (prior to post-surgical orthodontic treatment). Preoperative CT and stereolithography (STL) data that reflect the dental arch scan were superimposed to frame a composite three-dimensional (3D) skull model for a precise rendition of the bone and dentition.

Following the comprehensive 3D evaluation, virtual surgical planning and simulations were conducted using Mimics (version 17.0; Materialise, Leuven, Belgium). Osteoplasty of the inferior border of the mandible was simulated to achieve facial symmetry and coordination in the lower face. The final surgical plan from the STL was exported to the Ci-Navi system. Osteotomy guide plates, which were temporarily fixed on the anterior wall of the maxilla for surgical guidance, and occlusal splints were designed and fabricated with a 3D printer (Perfactory P4K; EnvisionTEC, Dearborn, MI, USA).

Transfer of the virtual plan during surgery using Ci-Navi

The designed 3D craniomaxillofacial model and original 3D model derived from preoperative CT data were both imported to the AccuNavi-A Surgical Navigation System (UEG Medical, Shanghai, China). Five to eight points were selected at the same position (frontal bone and zygomatic bone) on the original and the designed 3D model, and the 'superimposition' key in the software was selected. The original 3D model was then automatically superimposed onto the final 3D surgical simulation model using the surface-best-fit method. The superimposition accuracy was assessed by checking the degree of overlapping of the skull, frontal, and zygomatic bone contours in the two 3D models. 
Following patient-to-image registration, the navigation system automatically calculated the exact position of the tip of the navigation probe placed on a specific anatomical landmark in the patient space, which was subsequently transferred to the identical position in the CT image space (Figure 1).

Bilateral sagittal split ramus osteotomy of the mandible was performed first. A Le Fort 1 osteotomy was performed using osteotomy guide plates (Figure 2). Subsequently, the mobilized maxilla and distal mandibular segments were fastened to the occlusal splint and combined to form the maxillomandibular complex (MMC). The MMC was manually repositioned to the planned location under the guidance of the navigation probe as shown on the navigation station screen (Figure 3 ). The maxilla was rigidly fixed with four titanium plates placed bilaterally at the piriform rim and zygomatic buttress. The sections of the mandible were joined using titanium plates and screws. After fixation of the maxilla and mandible, the accuracy was double-checked under the guidance of the navigation probe. The asymmetric area of the mandible was precisely modified under $\mathrm{Ci}$ Navi guidance according to the surgical plan. A specially assembled navigation probe with a disposable $20 \mathrm{~mL}$ syringe was used to guide the osteoplasty in specific areas, such as the mandibular angle where access is restricted for regular navigation probes (Figure 4).

\section{Quantitative analysis of postoperative outcomes}

Both the planned protocol and the postoperative CT data were imported to Mimics (Materialise). Surface-rendered hard-tissue representations subsequent to voxelbased superimposition on the cranial base before and after surgery (planned model and postoperative model) were obtained. The Frankfort horizontal plane (FHP), midfacial sagittal plane (MSP) (perpendicular to the FHP through the nasion and basion), and coronal plane (CP) (perpendicular to the FHP through the sella) were selected as the three symmetry planes (Figure 5). Ten anatomical landmarks were selected (Table 1). For linear
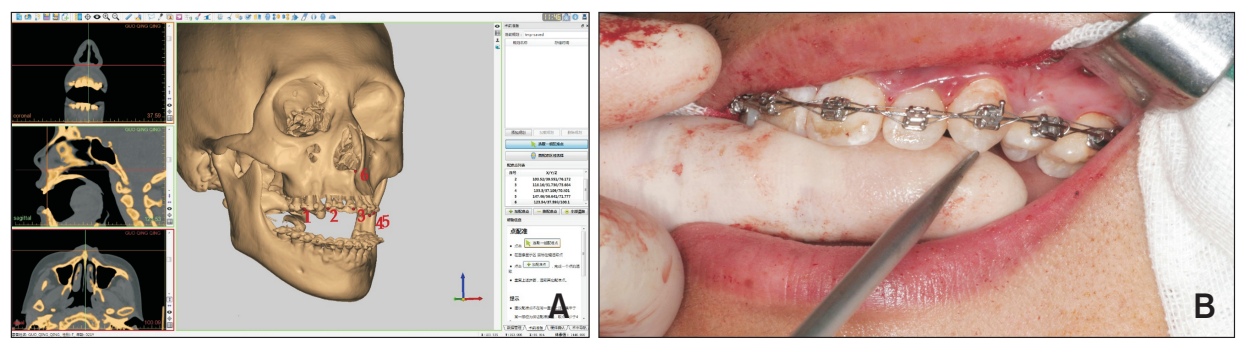

Figure 1. Registration of the navigation system. Registration landmarks were selected (A) and patient-to-image registration was performed (B).
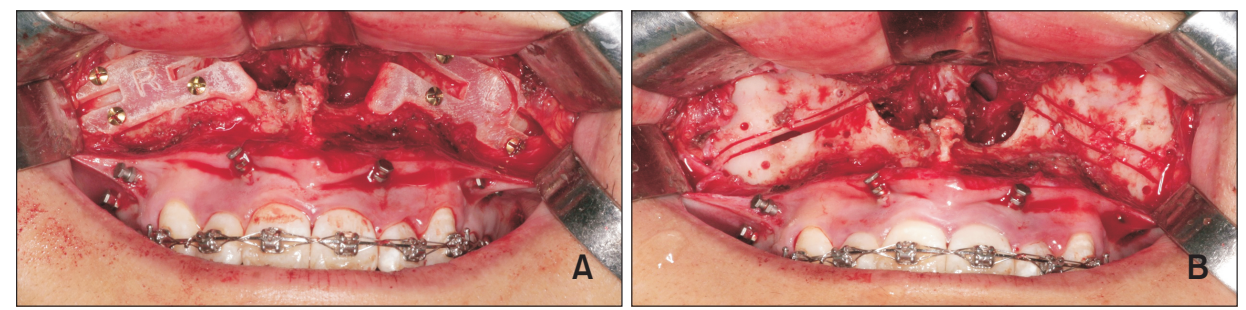

Figure 2. Le Fort I osteotomy. A, The osteotomy templates were fixed to the anterior wall of the maxilla using screws. B, A Le Fort I osteotomy was performed with guidance templates, and the osteotomy templates had been removed.
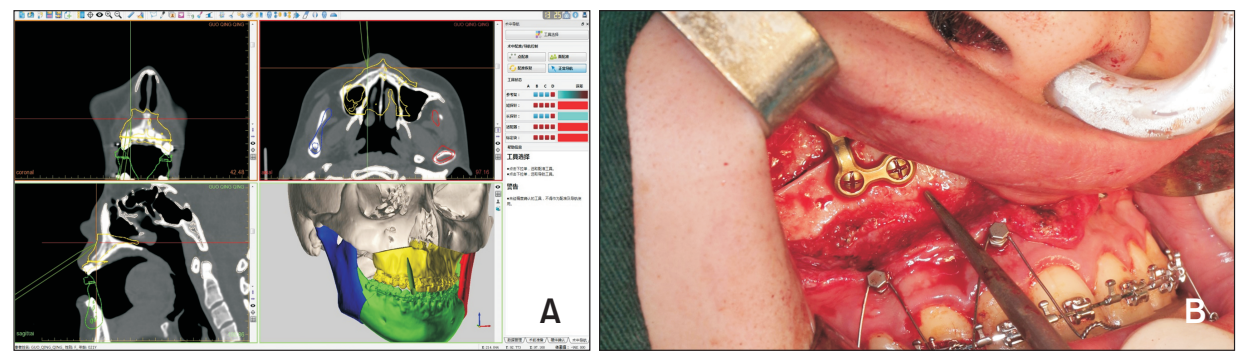

Figure 3. Repositioning of the maxillomandibular complex (MMC). A, Actual position of the MMC is displayed on the screen instantaneously. $\mathbf{B}$, Repositioning of the MMC during the operation under the guidance of a navigation probe. 

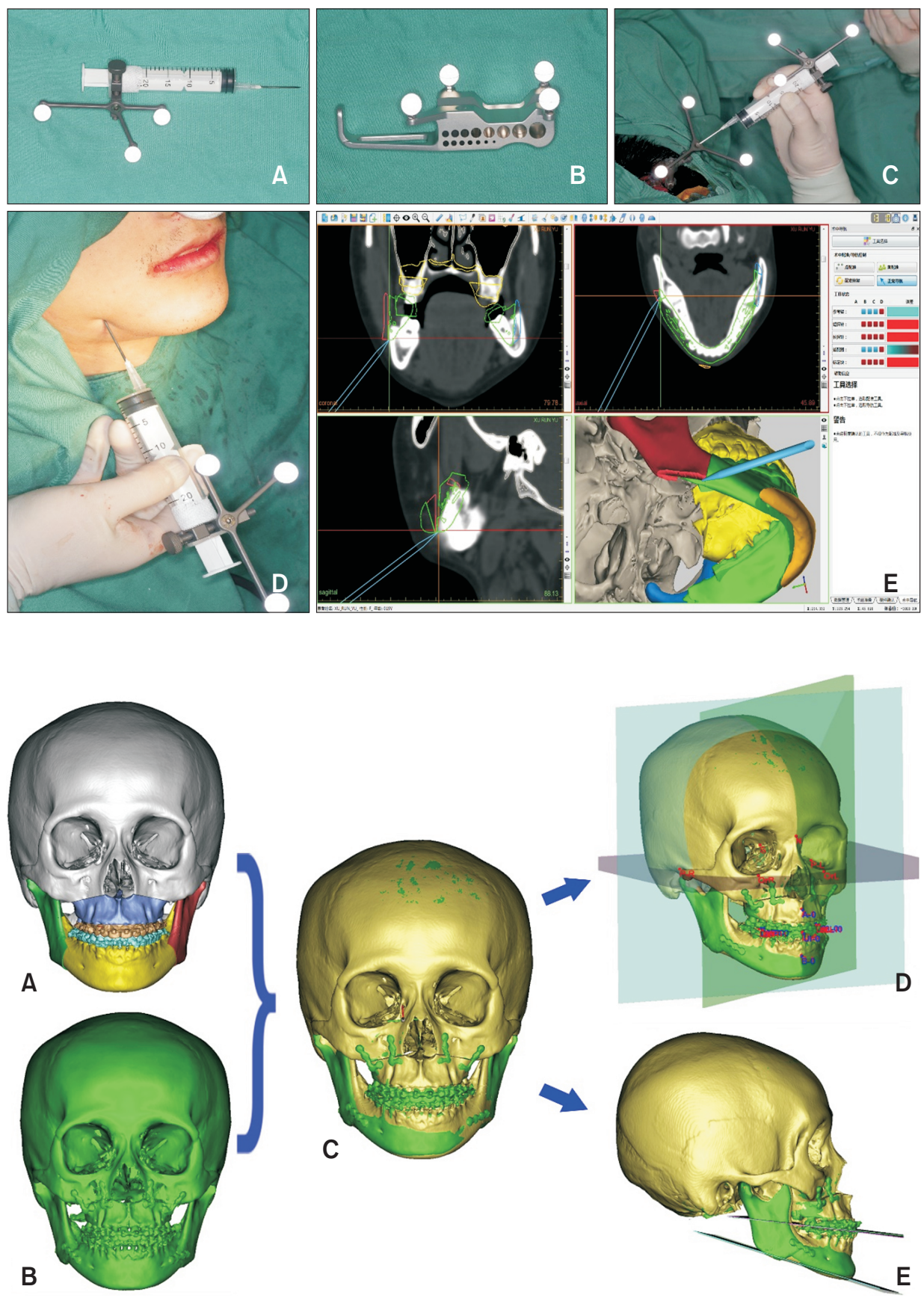

Figure 5. Evaluation. The preoperative plan (A) and postoperative outcome (B) were superimposed to construct a composite skull model (C). The linear and angular differences were measured using selected reference landmarks and planes (D, E).
Figure 4. Osteoplasty of the inferior border of the mandible. This procedure was performed under the guidance of specially assembled navigation probes, and the surgical outcome was displayed on the screen. A-C, Assembling and registering of the specialized navigation probe. D, The inferior border of the mandible was modified under the guidance of the navigation probe. E, The result was displayed on the screen. analysis, the distance from the selected landmarks to the three symmetry planes was gauged, and the differences between simulated and postoperative models were calculated. For angular analysis, the measurements of the angles formed by the occlusal and mandibular planes to the FHP and MSP were confirmed on the simulated and postoperative models, and the differences between both models were calculated.

\section{Statistical analysis}

The data were analyzed using SPSS 20.0 (IBM
Corp., Armonk, NY, USA). All data were normally distributed. To ensure interobserver reliability, the analysis was performed by two unified trained maxillofacial surgeons. To ensure intraobserver reliability, each observer recorded the measurements three times. Reliability was calculated using Pearson's intraclass correlation coefficient (ICC). The mean observation by two surgeons was considered final. Additionally, a paired $t$-test was used to calculate the linear and angular differences between the postoperative and planned outcomes. Statistical significance was specified 
Table 1. Linear differences $(\mathrm{mm})$ and angular differences $\left({ }^{\circ}\right)$ between preoperative planning $\left(\mathrm{T}_{0}\right)$ and postoperative outcome $\left(T_{1}\right)$ in the three-dimensional coordinate system

\begin{tabular}{|c|c|c|c|}
\hline \multirow{2}{*}{ Landmarks and symmetry planes } & \multicolumn{3}{|c|}{$\mathbf{T}_{1}-\mathbf{T}_{0}$} \\
\hline & FHP & MSP & $\mathbf{C P}$ \\
\hline $\mathrm{A}(\mathrm{mm})$ & $-0.71 \pm 0.58$ & $0.58 \pm 0.59$ & $-0.82 \pm 1.15$ \\
\hline $\mathrm{B}(\mathrm{mm})$ & $1.12 \pm 0.84$ & $0.80 \pm 0.65$ & $1.28 \pm 1.74$ \\
\hline $\mathrm{UI}(\mathrm{mm})$ & $0.79 \pm 0.47$ & $0.48 \pm 0.36$ & $0.82 \pm 0.44$ \\
\hline $\mathrm{LI}(\mathrm{mm})$ & $0.82 \pm 0.26$ & $0.76 \pm 0.59$ & $0.90 \pm 0.35$ \\
\hline $\mathrm{U} 6(\mathrm{~L})(\mathrm{mm})$ & $0.85 \pm 0.45$ & $-0.56 \pm 0.23$ & $0.88 \pm 0.29$ \\
\hline $\mathrm{U} 6(\mathrm{R})(\mathrm{mm})$ & $0.78 \pm 0.49$ & $0.75 \pm 0.23$ & $0.74 \pm 0.22$ \\
\hline $\mathrm{L} 6(\mathrm{~L})(\mathrm{mm})$ & $1.02 \pm 0.76$ & $0.63 \pm 0.31$ & $1.14 \pm 0.72$ \\
\hline $\mathrm{L} 6(\mathrm{R})(\mathrm{mm})$ & $1.07 \pm 0.61$ & $0.76 \pm 0.53$ & $1.18 \pm 0.77$ \\
\hline Co $(\mathrm{L})(\mathrm{mm})$ & $0.42 \pm 0.31$ & $0.57 \pm 0.35$ & $0.86 \pm 0.42$ \\
\hline $\mathrm{Co}(\mathrm{R})(\mathrm{mm})$ & $0.36 \pm 0.22$ & $0.49 \pm 0.21$ & $0.75 \pm 0.33$ \\
\hline Occlusion plane $\left(^{\circ}\right)$ & $1.07 \pm 1.54$ & $1.16 \pm 0.87$ & - \\
\hline Mandibular plane $\left(^{\circ}\right)$ & $1.22 \pm 0.97$ & $1.35 \pm 1.21$ & - \\
\hline
\end{tabular}

Values are presented as mean \pm standard deviation.

Paired $t$-test was performed, and no significant difference was found.

FHP, Frankfort horizontal plane; MSP, midsagittal plane; CP, coronal plane; A, subspinale; B, supramental; UI and LI, midpoint of the contact of the maxillary and mandibular central incisors, respectively; L, left; R, right; U6 and L6, mesiobuccal cusp of the maxillary and mandibular first molars, respectively; Co, the most superior point of condyle.

at $p<0.05$. The distribution difference of the upper incisor (UI) between the surgical plan and postoperative outcome was determined.

\section{RESULTS}

The mean intraobserver (0.96) and interobserver (0.92) ICC reliabilities were determined to be high. All correlations were highly significant $(p=0.002)$.

Linear differences between the preoperative plan and the final postoperative outcome were evaluated; there were no significant differences observed in any of the variables (Table 1). The overall mean difference in the distance between the landmarks to the FHP, MSP, and $\mathrm{CP}$ was $0.79 \mathrm{~mm}$. The mean distance between the maxillary landmarks, and the FHP, MSP, and CP was 0.62 $\mathrm{mm}$, and that between the mandibular landmarks and the symmetric planes was $0.88 \mathrm{~mm}$. The general mean linear difference in the distance between both the maxillary and mandibular landmarks, and the FHP, MSP, and CP were $0.91 \mathrm{~mm}, 0.52 \mathrm{~mm}$, and $1.19 \mathrm{~mm}$, respectively. The maxillary deviation was smaller than that of the mandible $(0.62$ vs. $0.88 \mathrm{~mm})$. The results also showed a lesser deviation of the landmarks relative to the MSP $(0.52 \mathrm{~mm})$ than the FHP $(0.91 \mathrm{~mm})$ and CP $(1.19 \mathrm{~mm})$. The most superior point of the condyle did not significantly differ between the postoperative measurement and the preoperative plan; however, the difference in the anterior-posterior direction was larger than that in the horizontal and vertical directions.

The angular difference in the occlusal and mandibular planes between the surgical plan and the actual postoperative outcome is depicted in Table 1. The overall mean angular difference was $1.20^{\circ}$.

The absolute mean difference in the $\mathrm{Ul}$ between the surgical plan and the postoperative outcome was $<1$ $\mathrm{mm}$ with respect to the FHP, MSP, and CP in 40 cases.

\section{DISCUSSION}

This study compared preoperative planning and postoperative outcome to examine the accuracy of Ci-Navi in bimaxillary orthognathic surgery. We found that CiNavi was a reliable method for the accurate transfer of the surgical plan during an operation.

The success of orthognathic surgery is dependent on both the surgical technique and accurate surgical planning. ${ }^{1,2}$ The advantage of using CASP in the treatment planning of complex craniomaxillofacial deformities has been confirmed. ${ }^{2,16} \mathrm{Lin}$ and $\mathrm{Lo}^{15}$ conducted a literature review of the application of 3D computer-assisted surgical simulation and Ci-Navi in orthognathic surgery during a 10-year period, and concluded that the application of computer-assisted techniques in orthognathic surgery has the merits of optimal functional and esthetic outcomes, patient satisfaction, accurate interpretation of 
the treatment plan, and facilitation of intraoperative manipulation.

A computerized treatment plan is trivial if it is not reproducible in the patient. ${ }^{16}$ Therefore, the accurate transfer of the surgical plan during the operation is of paramount importance. However, it is often impossible to determine whether the surgical plan is accurately transferred during the operation. Before the application of Ci-Navi, maxilla repositioning was typically performed using an intermediate occlusal splint. Manual surgery is time-consuming and laborious, and the accuracy is poor. Relying on the traditional intermediate splint and intraoperative measurement for surgical guidance confounds the advantages of 3D technology. ${ }^{10}$ Despite, recent improvement in surgical plan transfer through maxillary reposition templates (integral or modular) ${ }^{7,9-11}$ and the development of a device to assist in the maintenance of the condyle position, ${ }^{10}$ the design of these surgical templates is cumbersome, and a certain thickness and hard texture of the maxilla is required for their application. Osteotomy guide plates were applied in our study because they can rapidly indicate the osteotomy line of the maxilla, particularly in cases requiring the removal of redundant bone. Moreover, the contact between bone sections will be more extensive, thus increasing initial stability and reducing destabilization.

Ci-Navi has been increasingly applied as an effective tool for treating craniomaxillofacial malformations, ${ }^{17}$ including those associated with facial asymmetry. ${ }^{18} \mathrm{Sev}$ eral computer-aided surgical navigation systems have become commercially available in the field of craniomaxillofacial surgery. Stryker (Stryker-Leibinger, Kalamazoo, Ml, USA) and VectorVision (BrainLab AG, Munich, Germany) are two commonly used navigation systems that have been extensively studied. Tsuji et al. ${ }^{19}$ reported an optical tracking system for navigation using chargedcoupled-device video cameras and light-emitting diodes that provided sufficient accuracy. However, the application of this method has rarely been reported. Mazzoni et al. ${ }^{20}$ demonstrated the improved accuracy of reproducibility with the use of the eNlite Navigation System by Stryker for guidance. Bell ${ }^{21}$ found this navigation system particularly useful for the correction of facial asymmetry and hemimandibular hyperplasia. Shim et al. ${ }^{22}$ also reported that surgeons can accurately carry out the preoperative plan with regard to the position of the instrument, which can be visualized with the Stryker system. Zinser et al. ${ }^{23}$ compared the use of the VectorVision navigation system (BrainLab AG), computer aided design/computer aided manufacturing (CAD/CAM) splints, and classic intermaxillary splints in orthognathic surgery, and found that the CAD/CAM splint and the navigation system had a higher accuracy than the intermaxillary splints. Han et al. ${ }^{24}$ reported that for hemimandibular hyperplasia correction by simultaneous orthognathic surgery and condylectomy, surgical results can be validated during surgery under the guidance of the VectorVision navigation system. Sun et al. ${ }^{25}$ reported that the Kolibri navigation system (BrainLab $A G$ ) is a promising tool for measuring the postoperative change in the maxilla in bimaxillary surgery.

These navigation systems allow surgeons to determine the precise location of bone landmarks with a precision of 1-2 mm. ${ }^{26}$ With real-time navigation guidance, more accurate and reliable repositioning of the downfractured maxilla can be achieved during orthognathic surgery. ${ }^{27}$ In the present study, the general mean linear distinction was $0.79 \mathrm{~mm}$, and the general mean angular difference was $1.20^{\circ}$. Ci-Navi was more favorable for the maxilla than the mandible (a mean linear distinction of 0.62 vs. $0.88 \mathrm{~mm}$ ). It was also observed that the deviation from the MSP $(0.52 \mathrm{~mm})$ was less than that from the FHP $(0.91 \mathrm{~mm})$ and the CP $(1.12 \mathrm{~mm})$, indicating that Ci-Navi works better in the midline. The AccuNavA surgical navigation system performed well. However, it is worth noting that the difference in the mandible was larger than that in the maxilla, particularly for mesiobuccal cusp of the first mandibular molar. This may be because the base of the skull is the center of rotation. While the maxilla is close to the base of the skull, the mandible is far from it. Therefore, even a tiny movement in the maxilla extends to the mandible and amplifies the effect, causing a larger change in the mandible. This is consistent with the findings of Xia et al. ${ }^{28}$

The surgical accuracy of the UI is critical for esthetics. A minor inaccuracy in the position of the UI, particularly in the vertical and sagittal directions, can affect patient satisfaction. The absolute mean difference in the UI compared to the measurement determined through the navigation system and the actual surgical change was $<1 \mathrm{~mm}$ in the three directions in 40 cases. This is in agreement with the findings of Sun et al..$^{25}$ The utilization of Ci-Navi resulted in an excellent $\mathrm{Ul}$ outcome.

Augmented reality is another method for controlling maxillary translocation by allowing the real-time visual tracing of anatomical structures in superimposition with volume-rendered CT or magnetic resonance imaging. ${ }^{23,29}$ The virtual plan and real position of the maxilla could be superimposed during the surgery using a video graphics array camera. Maxillary positioning was accomplished to within $1 \mathrm{~mm}$. However, based on the nature of manipulation, manual repositioning of the maxilla using a handheld display is particularly challenging. ${ }^{23}$

With the use of Ci-Navi, repositioning of the proximal fragment of the mandible is performed under guidance to ensure that there are no apparent roll, yaw, and pitch movements in the mandibular angle area until the condyle is seated in the correct position. In the traditional 
method, the repositioning is mainly dependent on the experience and observation of the surgeon, which is subjective and a calculated estimation. Despite meticulous planning, residual asymmetry is common and mandibular osteoplasty is a requisite. It has been reported that 34\% of cases with dentomaxillofacial deformity had asymmetry. ${ }^{30}$ In our study, the proportion was larger (23 of $45,51 \%)$. Osteoplasty of the inferior border of the mandible was conducted in 25 cases. Ci-Navi is particularly useful in correlating facial asymmetry with hemimandibular hyperplasia or atrophy. Osteoplasty of the inferior border of the mandible when conducted under navigation guidance is able to achieve the most accurate vertical and horizontal relationship with symmetry and coordination in the lower face profile. ${ }^{21}$

Moreover, the limited operating space in the oral cavity made it difficult to access the middle and rear parts of the inferior border of the mandible with the use of a conventional navigation probe. The specially assembled 'navigation probe' with a sharp needle tip enabled penetration of the skin to reach the cortical bone surface without leaving a scar. This needle can thus be used for osteoplasty in areas inaccessible to routine navigation probes.

There are some limitations to this technique. The surgical preparation using the navigation system can prolong the operation time (by approximately $20 \mathrm{~min}$ utes), which requires improvement. A $1 \mathrm{~cm}$ incision is needed behind the hairline on the scalp for navigation reference frame fixation. Moreover, 3D planning and navigation increase the cost of surgery for every patient (approximately by 10\%). Furthermore, the accuracy of the operation varies when different virtual plan transfer methods are used. Therefore, the advantages and limitations of these methods should be further investigated. If randomized controlled study can be done in the future, it will make the study more systematic and complete.

\section{CONCLUSION}

The use of Ci-Navi demonstrated $0.79 \mathrm{~mm}$ of overall mean difference in the distance between the landmarks. Based on the results of this study, Ci-Navi may be a reliable method for facilitating accurate transfer of the surgical plan during bimaxillary orthognathic surgery.

\section{CONFLICTS OF INTEREST}

No potential conflict of interest relevant to this article was reported.

\section{REFERENCES}

1. Xia JJ, Gateno J, Teichgraeber JF, Christensen AM,
Lasky RE, Lemoine JJ, et al. Accuracy of the computer-aided surgical simulation (CASS) system in the treatment of patients with complex craniomaxillofacial deformity: a pilot study. J Oral Maxillofac Surg 2007;65:248-54.

2. Xia JJ, Gateno J, Teichgraeber JF. New clinical protocol to evaluate craniomaxillofacial deformity and plan surgical correction. J Oral Maxillofac Surg 2009;67:2093-106.

3. Hsu SS, Gateno J, Bell RB, Hirsch DL, Markiewicz $\mathrm{MR}$, Teichgraeber JF, et al. Accuracy of a computeraided surgical simulation protocol for orthognathic surgery: a prospective multicenter study. J Oral Maxillofac Surg 2013;71:128-42.

4. Gateno J, Xia JJ, Teichgraeber JF. New 3-dimensional cephalometric analysis for orthognathic surgery. J Oral Maxillofac Surg 2011;69:606-22.

5. Sharifi A, Jones R, Ayoub A, Moos K, Walker F, Khambay B, et al. How accurate is model planning for orthognathic surgery? Int J Oral Maxillofac Surg 2008;37:1089-93.

6. Xia J, Ip HH, Samman N, Wang D, Kot CS, Yeung RW, et al. Computer-assisted three-dimensional surgical planning and simulation: 3D virtual osteotomy. Int J Oral Maxillofac Surg 2000;29:11-7.

7. Zinser MJ, Mischkowski RA, Sailer HF, Zöller JE. Computer-assisted orthognathic surgery: feasibility study using multiple CAD/CAM surgical splints. Oral Surg Oral Med Oral Pathol Oral Radiol 2012;113: 673-87.

8. Tran NH, Tantidhnazet S, Raocharernporn S, Kiattavornchareon S, Pairuchvej V, Wongsirichat N. Accuracy of three-dimensional planning in surgeryfirst orthognathic surgery: planning versus outcome. J Clin Med Res 2018;10:429-36.

9. Zhang N, Liu S, Hu Z, Hu J, Zhu S, Li Y. Accuracy of virtual surgical planning in two-jaw orthognathic surgery: comparison of planned and actual results. Oral Surg Oral Med Oral Pathol Oral Radiol 2016; 122:143-51.

10. Polley JW, Figueroa AA. Orthognathic positioning system: intraoperative system to transfer virtual surgical plan to operating field during orthognathic surgery. J Oral Maxillofac Surg 2013;71:911-20.

11. Li B, Zhang L, Sun H, Yuan J, Shen SG, Wang X. A novel method of computer aided orthognathic surgery using individual CAD/CAM templates: a combination of osteotomy and repositioning guides. $\mathrm{Br} \mathrm{J}$ Oral Maxillofac Surg 2013;51:e239-44.

12. Baumann A, Schicho K, Klug C, Wagner A, Ewers R. Computer-assisted navigational surgery in oral and maxillofacial surgery. Atlas Oral Maxillofac Surg Clin North Am 2005;13:41-9.

13. Ewers R, Schicho K, Undt G, Wanschitz F, Truppe 
$M$, Seemann R, et al. Basic research and 12 years of clinical experience in computer-assisted navigation technology: a review. Int J Oral Maxillofac Surg 2005;34:1-8.

14. Stokbro K, Aagaard E, Torkov P, Bell RB, Thygesen T. Virtual planning in orthognathic surgery. Int $\mathrm{J}$ Oral Maxillofac Surg 2014;43:957-65.

15. Lin HH, Lo LJ. Three-dimensional computer-assisted surgical simulation and intraoperative navigation in orthognathic surgery: a literature review. J Formos Med Assoc 2015;114:300-7.

16. Xia JJ, Gateno J, Teichgraeber JF. Three-dimensional computer-aided surgical simulation for maxillofacial surgery. Atlas Oral Maxillofac Surg Clin North Am 2005;13:25-39.

17. Yu H, Shen SG, Wang X, Zhang L, Zhang S. The indication and application of computer-assisted navigation in oral and maxillofacial surgery-Shanghai's experience based on 104 cases. J Craniomaxillofac Surg 2013;41:770-4.

18. Sadiq Z, Collyer J, Sneddon K, Walsh S. Orthognathic treatment of asymmetry: two cases of "waferless" stereotactic maxillary positioning. $\mathrm{Br} \mathrm{J}$ Oral Maxillofac Surg 2012;50:e27-9.

19. Tsuji M, Noguchi N, Shigematsu M, Yamashita Y, lhara K, Shikimori M, et al. A new navigation system based on cephalograms and dental casts for oral and maxillofacial surgery. Int J Oral Maxillofac Surg 2006;35:828-36.

20. Mazzoni S, Badiali G, Lancellotti L, Babbi L, Bianchi A, Marchetti C. Simulation-guided navigation: a new approach to improve intraoperative threedimensional reproducibility during orthognathic surgery. J Craniofac Surg 2010;21:1698-705.

21. Bell RB. Computer planning and intraoperative navigation in orthognathic surgery. J Oral Maxillofac Surg 2011;69:592-605.

22. Shim BK, Shin HS, Nam SM, Kim YB. Real-time navigation-assisted orthognathic surgery. J Craniofac
Surg 2013;24:221-5.

23. Zinser MJ, Mischkowski RA, Dreiseidler T, Thamm OC, Rothamel D, Zöller JE. Computer-assisted orthognathic surgery: waferless maxillary positioning, versatility, and accuracy of an image-guided visualisation display. Br J Oral Maxillofac Surg 2013; 51:827-33.

24. Han B, Wang X, Li Z, Yi B, Liang C, Wang X. Hemimandibular hyperplasia correction by simultaneous orthognathic surgery and condylectomy under digital guidance. J Oral Maxillofac Surg 2018;76:1563. e1-1563.e18.

25. Sun Y, Luebbers HT, Agbaje J0, Lambrichts 1, Politis $C$. The accuracy of image-guided navigation for maxillary positioning in bimaxillary surgery. $\mathrm{J}$ Craniofac Surg 2014;25:1095-9.

26. Luebbers HT, Messmer P, Obwegeser JA, Zwahlen RA, Kikinis R, Graetz KW, et al. Comparison of different registration methods for surgical navigation in cranio-maxillofacial surgery. J Craniomaxillofac Surg 2008;36:109-16.

27. Ong TK, Banks RJ, Hildreth AJ. Surgical accuracy in Le Fort 1 maxillary osteotomies. Br J Oral Maxillofac Surg 2001;39:96-102.

28. Xia JJ, Shevchenko L, Gateno J, Teichgraeber JF, Taylor TD, Lasky RE, et al. Outcome study of computer-aided surgical simulation in the treatment of patients with craniomaxillofacial deformities. J Oral Maxillofac Surg 2011;69:2014-24.

29. Mischkowski RA, Zinser MJ, Kübler AC, Krug B, Seifert U, Zöller JE. Application of an augmented reality tool for maxillary positioning in orthognathic surgery - a feasibility study. J Craniomaxillofac Surg 2006;34:478-83.

30. Severt TR, Proffit WR. The prevalence of facial asymmetry in the dentofacial deformities population at the University of North Carolina. Int J Adult Orthodon Orthognath Surg 1997;12:171-6. 